\title{
Diabetes in Sub-Saharan Africa - Overview of a Looming Health Challenge
}

\author{
Felix K Assah¹ and Jean-Claude Mbanya ${ }^{2}$
}

1. Doctoral Student, MRC Epidemiology Unit, Institute of Metabolic Science, Addenbrooke's Hospital, Cambridge; 2. Professor of Medicine and Endocrinology, Department of Internal Medicine and Specialities, Faculty of Medicine and Biomedical Sciences, University of Yaoundé I

DOI: $10.17925 / E E .2009 .05 .00 .13$

\begin{abstract}
The number of people living with diabetes in the world is increasing rapidly. Most of the increase will occur in developing countries such as those in sub-Saharan Africa. Diabetes is currently recognised as a real and imminent threat to social and economic development globally and is set to be a major public health challenge in the 21st century. In sub-Saharan Africa, the challenge posed by diabetes is even more overwhelming since diabetes will have to share scarce resources with infections and malnutrition. Even worse, diabetes still has to gain sufficient political and social recognition in order to warrant aggressive national policies for prevention and treatment. In this article we present an overview of the burden of diabetes in sub-Saharan Africa and the specificities of care and control, and highlight the importance of developing effective national diabetes programmes.
\end{abstract}

\section{Keywords}

Diabetes, sub-Saharan Africa, epidemiology, morbidity, mortality, cost of diabetes care, diabetes control programme

Disclosure: The authors have no conflicts of interest to declare.

Received: 4 May 2009 Accepted: 16 July 2009

Correspondence: Felix K Assah, MRC Epidemiology Unit, Institute of Metabolic Science, Box 285, Addenbrooke's Hospital, Hills Road, Cambridge CB2 0QQ, UK.

E: felix.assah@mrc-epid.cam.ac.uk

A few decades ago, diabetes was considered a disease of affluent societies. This statement would be rather inappropriate today as it is known that most of the world's diabetes patients live in developing countries. In absolute numbers, most of these people live in China and India. However, the burden of diabetes in sub-Saharan Africa (SSA) is already substantial, and continues to increase at a very rapid rate: current predictions estimate that the number of people living with diabetes on the continent will almost double over the next 20 years. ${ }^{1}$ This is not good news for a continent that has been the hardest hit by the HIV/AIDS pandemic and that currently is home to about $70 \%$ of the billion poorest people of the world. ${ }^{2}$

The increasing prevalence of diabetes and other non-communicable diseases in Africa is a result of an epidemiological transition driven by rapid urbanisation of communities. Urbanisation brings with it a nutritional transition from traditional natural foods to processed energy-dense foods, as well as a substantial reduction in physical activity levels due to mechanisation of work and transport. These changes have led to an increasing level of obesity in young adults and adults, which is driving the surge in diabetes. The epidemiological transition Africa is currently undergoing is not exactly the same as that which most western countries went through: the transition in Africa is following a delayed model ${ }^{3}$ characterised by high fertility and high but reducing mortality, resulting in a double demographic disease pattern. This means that the low-income economies of most African countries and their strained healthcare systems have to grapple with the burden of infections, underweight and chronic malnutrition in children and obesity and cardiovascular diseases in adults.

\section{Prevalence, Risk Factors and Trends}

There is a dearth of good-quality epidemiological data on diabetes prevalence, incidence and trends on the African continent. The difficulty is even more critical when trying to extricate the separate rates of type 1 or type 2 diabetes. Only a handful of studies have described the epidemiology of type 1 diabetes in an African population, and of these only a couple are from SSA. ${ }^{4}$ Due to limitations in study methods and design, many of these studies do not provide reliable population estimates, in addition to the fact that these estimates may include cases of malnutrition-related diabetes or ketosis-prone diabetes $^{5}$ due to diagnostic constraints. Notwithstanding, the International Diabetes Federation (IDF) estimates that there were a total of almost 38,000 prevalent cases of type 1 diabetes in SSA in 2007, which was higher than those in South and Central America or the Western Pacific region of the IDF despite the fact that these regions had a much higher denominator population than SSA. ${ }^{1}$ The peak age of occurrence of type 1 diabetes has been reported to be much later in Africans (22-23 years) compared with Caucasians (12-13 years). ${ }^{6}$ Current estimates of the prevalence of type 2 diabetes in Africa from the IDF ${ }^{1}$ are based on data from only a handful of countries. In 2007, there were an estimated 10.4 million people living with diabetes in Africa, with this number expected to increase to 18.7 million by 2025 an $80 \%$ increase (see Figure 1). This projection may be a conservative estimate, even considering the effect HIV/AIDS is going to have on reducing the size of the adult population that is at greatest risk of developing diabetes, since with increasing access to antiretroviral chemotherapy the region would probably witness an increase in people developing antiretroviral therapy (ART)-induced glucose intolerance. Our data in Cameroonian adults based on two cross- 
Figure 1: Prevalence of Diabetes in 2007 and Projections for 2025, with Projected Per Cent Changes

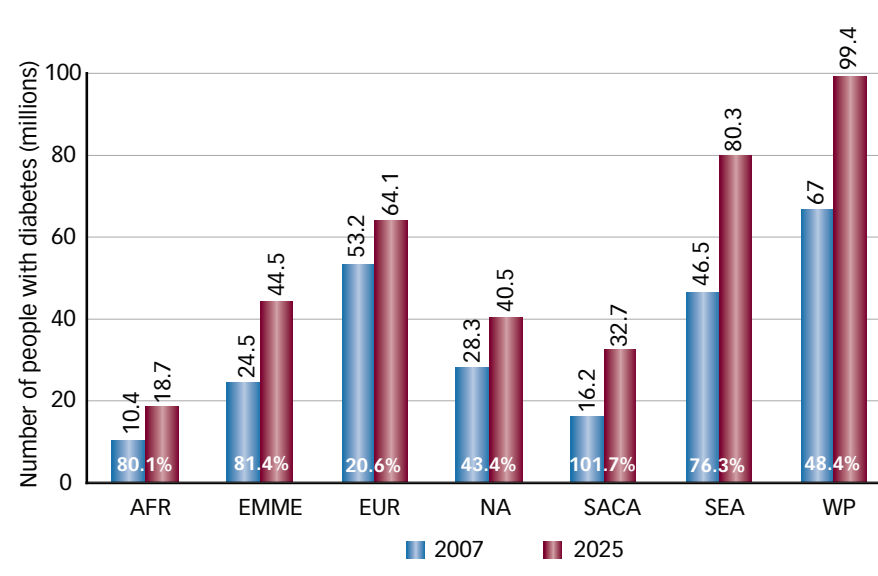

Source: IDF Diabetes Atlas. ${ }^{\top}$ AFR $=$ Africa; $E M M E=$ Eastern Mediterranean and Middle East; EUR = Europe; $N A=$ North America; SACA = South and Central America; SEA = South-East Asia; $W P=$ Western Pacific.

Figure 2: Prevalence of Type 2 Diabetes in Cameroon, 1994-2003

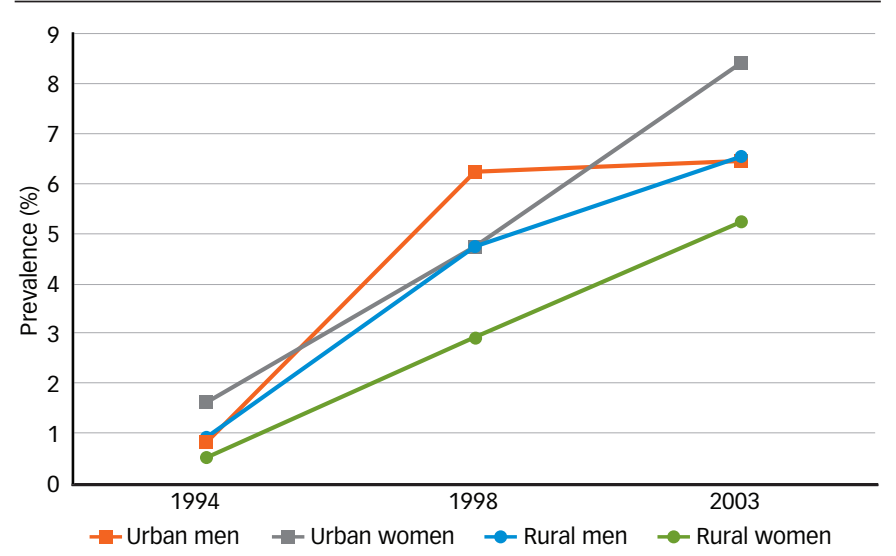

Prevalence standardised according to new world population distribution.

sectional surveys over a 10-year period (1994-2004) revealed an almost 10 -fold increase in diabetes prevalence (see Figure 2). ${ }^{7}$ Most of the classic risk factors have been reported to be associated with diabetes in Africans. However, a lot of these papers are based on empirical evidence or poorly measured lifestyle exposure. Urbanisation, resulting in increased levels of physical inactivity and high-fat diets, has been described as the major driver of increasing levels of obesity and diabetes. Many studies have shown a distinct rural-urban increased risk of obesity and diabetes, ${ }^{8,9}$ with a lengthier stay in the urban environment conferring a higher risk..$^{10,11}$ Even though the idea of lower physical activity in urban areas compared with rural areas is intuitively plausible, most of the studies reporting physical activity levels have used very crude questions to obtain these data. A few studies have used validated physical activity questionnaires to demonstrate this urbanisation gradient, ${ }^{12,13}$ as well as the increased risk of diabetes associated with reduced physical activity levels. ${ }^{14}$ There are no studies that have used objective assessment of physical activity; neither are there any studies that have documented temporal trends of physical activity levels in communities using comparable methids. The adoption and use of the World Health Organization (WHO) STEPS Global Physical Activity Questionnaire for surveillance of habitual physical activity by many SSA countries ${ }^{15}$ will hopefully provide some answers in the near future about trends in population levels of physical activity, despite the limitations of self-reported physical activity.
The difficulty of assessing diet and nutritional data in free-living individuals makes this as challenging as collecting physical activity data in many resource-limited countries. Studies in South African blacks have shown a trend of increasing consumption of fat and decreasing consumption of carbohydrates in both urban and rural areas. ${ }^{16}$ However, the notion of increased consumption of fat as a marker of urbanisation and westernisation of the African diet was not confirmed in our own studies, in which we found the highest consumption of fat in rural Cameroonians compared with urban Cameroonians, black Jamaicans and blacks in Manchester. ${ }^{17}$ We postulated that the excess consumption of fats in the rural area is more than compensated for by very high levels of physical activity. Whatever the interplay in the lifestyle factors, there has clearly been a remarkable increase in the prevalence of obesity in many African communities. Abubakari et al. ${ }^{18}$ pooled data from studies in west African adults and showed that the prevalence of obesity has more than doubled in urban regions from 7 to $15 \%$ over a 15-year period from 1990 to 2004. Women and urban dwellers had a significantly higher prevalence of obesity compared with men and rural dwellers, respectively. Similar results have been reported in other African populations. ${ }^{16,19}$ Data from repeated surveys in the same areas in Cameroon over a 10-year period showed an overall increase in prevalence of overweight and obesity in the rural site (increase of 54\% in women and $82 \%$ in men) and of central obesity in the urban site (increase of $32 \%$ in women and $190 \%$ in men). ${ }^{20}$ The public health challenge posed by obesity in these populations is far from over as excess weight is perceived positively as a sign of wealth, with a positive association between socioeconomic status and obesity seen in developing countries in contrast to the negative association in developed countries. The stigma associated with HIV-related weight loss only further aggravates the social perception of slim body size.

The prevalence of diabetes increases with age. However, the majority of people living with diabetes in SSA countries are in the 45-64 year age group, in contrast to developed countries, where the highest prevalence of diabetes is in those 65 years of age and above ${ }^{21}$ (see Figure 3). This underscores the point that the economic burden of diabetes in developing countries will be compounded by its effect on the working age group.

\section{Morbidity and Mortality}

SSA is currently struggling under a multiple disease burden that is not really getting lighter over the years. The emerging and increasing prevalence of obesity, diabetes and non-communicable diseases is not accompanied by any marked improvements in infections or undernutrition; many studies have reported high rates of co-existence of obesity and undernutrition in the same communities and even in the same households. ${ }^{22}$ This means that diabetes has to compete for scarce financial and healthcare resources with infections and undernutrition. Of course, this does not sound like a recipe for success. Prevalent undiagnosed diabetes is very high - about $80 \%$ in data from Cameroon. ${ }^{23}$ Even in known diabetes patients, control is often very poor. Only about one in four known diabetes patients in a population survey in Cameroon had optimal fasting blood glucose levels. ${ }^{23}$ In a study in Ethiopia of 105 diabetes patients seen by one physician, despite a low body mass index (BMI) - median BMl was $20.6 \mathrm{~kg} / \mathrm{m}^{2}$ - mean glycated haemoglobin $\left(\mathrm{HbA}_{1 \mathrm{C}}\right)$ was $11.3 \pm 2.8 \%$ and $68 \%$ of patients had an $\mathrm{HbA}_{1 \mathrm{C}}$ over $10 \%{ }^{24}$

Published data on acute complications of diabetes in SSA are scarce, but nonetheless point to the fact that these are more common than in 
developed countries and mostly carry a worse outcome. Case fatality rates for diabetic coma of $10-20 \%$ have been reported in studies in specialised hospitals. ${ }^{25,26}$ Evidently, the outcome of such cases in more deprived areas with less specialised hospitals would be much worse. These acute complications are usually made worse by accompanying severe infections, which are usually the precipitating factor. Toure et al. ${ }^{27}$ reported three-fold higher tuberculosis-related hospital mortality in diabetes patients compared with non-diabetes controls.

Chronic microvascular and neuropathic complications of diabetes have been reported to be highly prevalent across different populations in SSA. As summarised by Gill et al. ${ }^{28}$ the reported prevalence of the different chronic complications varies enormously between studies. Prevalence rates of retinopathy (15-55\%), nephropathy (32-57\%) and neuropathy (10-42\%), with a $49 \%$ prevalence of erectile dysfunction in men, have been reported across studies in SSA. ${ }^{29}$ These data have to be interpreted cautiously as there are wide differences in study design, population characteristics and other underlying risk factors. In many of these patients, complications are already present at the initial diagnosis of diabetes. Worse still, in some patients the initial diagnosis of diabetes is made during consultation or hospital admission for a diabetes-related complication in a hitherto unknown diabetes subject. Retinopathy is reported to be present in $21-25 \%$ of patients at diagnosis. ${ }^{29}$ In a study from Nigeria, $25 \%$ of patients admitted to hospital for diabetic foot were previously undiagnosed..$^{30}$ In another hospital audit from Cameroon, ${ }^{25} 21 \%$ of patients admitted in diabetic coma into the endocrinology unit of a main teaching hospital were not previously known to have diabetes.

Macrovascular complications of diabetes including coronary heart disease (CHD) and stroke appear to be less frequent in SSA than in other regions: ${ }^{31}$ in fact, blacks are at lower risk of macrovascular disease compared with other ethnic groups. Issues with data quality and absence of vital statistics may be involved in the information on these diseases from many SSA countries. However, in a study from South Africa in the same community and using the same methods, Kalk and Joffe $^{32}$ found in a sample of adults with diabetes that CHD was present in $4 \%$ of black Africans and in $23 \%$ of Caucasians.

\section{The Cost of Diabetes}

Healthcare in most of SSA is almost entirely privately purchased. Considering that the majority of the poorest people on Earth live in SSA, this statement intuitively signals disaster. A recent study by Kirigia et al. ${ }^{33}$ clearly demonstrated that the cost of diabetes care is going to be overwhelming for the poorest countries of the region. This study shows that while the direct cost of diabetes per person with diabetes is only a fraction ( $<25 \%$ ) of the gross national income (GNI) per capita for the 12 richest countries, the direct cost for the 34 poorest countries of the region is $125 \%$ of their GNI per capita (see Figure 4). For these poorest countries, the total cost (direct and indirect) of diabetes per person with diabetes is more than double the GNI per capita.

The few studies on cost of diabetes care (usually on small samples) that have been carried out in the region confirm the above estimates. Akoussou-Zinsou and Amedegnato ${ }^{34}$ reported in 2001 that the direct cost of diabetes care at a teaching hospital in Togo was US\$342 and US\$110 per person for 'complicated' and 'uncomplicated' diabetes patients, respectively. The estimated GNI per capita for Togo at the time was approximately US $\$ 385$. Chale et al. ${ }^{35}$ reported an average annual direct cost of diabetes care in Tanzania in 1989-1990 of US\$287 for a
Figure 3: Estimated Number of Adults with Diabetes in Sub-Saharan Africa and in Developed Countries by Age Group and Year
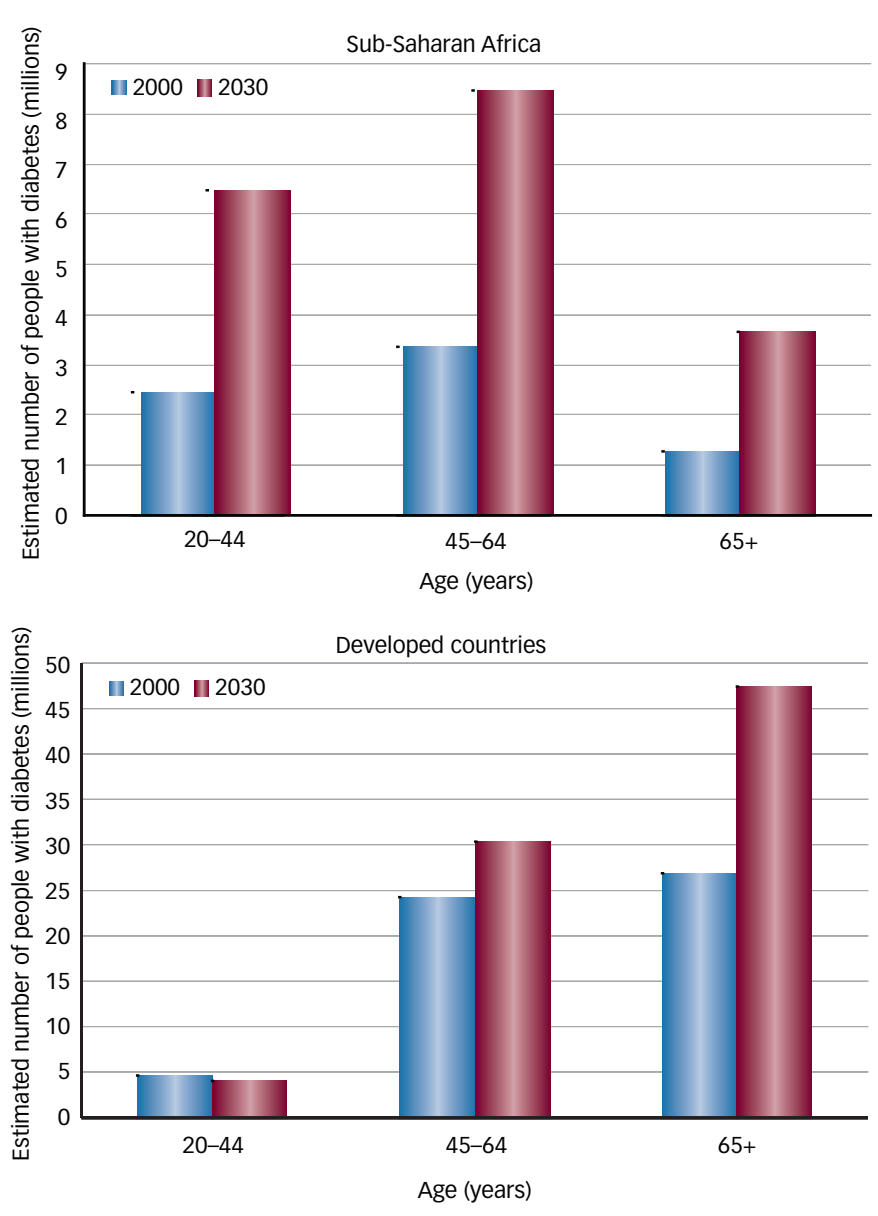

Source: Wild et al., 2004. ${ }^{21}$

Figure 4: Direct, Indirect and Total Cost of Diabetes per Person with Diabetes Compared with the Average Gross National Income Per Capita in the WHO Africa Region

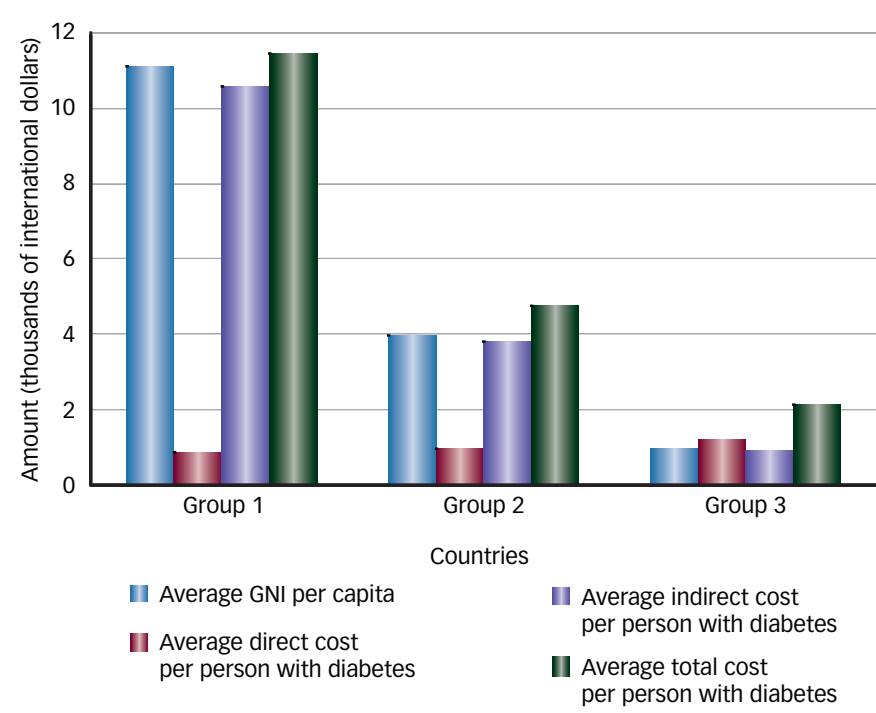

Countries are grouped according to average gross national income per capita. Source: Kirigia et al. ${ }^{33}$

patient requiring insulin and US\$103 for a patient not requiring insulin. In a study on type 1 diabetes patients in Sudan, Elrayah et al..$^{36}$ reported a partial direct cost of care for each child with type 1 diabetes of US $\$ 283$, 


\section{The Diabetes Declaration for Africa - Call to Action ${ }^{46}$}

The IDF-Africa, the WHO-AFRO and the African Union call on the governments of African countries, non-governmental organisations, international donor agencies, industry, healthcare providers and all partners in diabetes to ensure:

- adequate, appropriate and affordable medications and supplies for people living with diabetes;

- earlier detection of and optimal quality of care for diabetes;

- effective efforts to create healthier environments and to prevent diabetes;

- the identification and dissemination of information, education and communication to empower people with diabetes to access appropriate diabetes services and improve self care;

- equitable access to care and prevention services for people with or at risk of diabetes;
- awareness of diabetes in the community and among healthcare providers;

- a truly integrated approach that utilises the whole health workforce to address infectious and non-communicable diseases simultaneously;

- government commitment to reducing the personal and public health burden of diabetes; and

- partnerships and collaboration within and between government sectors, private sectors, non-government organisations and communities to create community and workplace environments that promote better health. in a country with a gross domestic product (GDP) per capita of US $\$ 300$ and a per capita government expenditure on health of US\$3.

These data undeniably indicate that the cost of diabetes care constitutes a huge burden on society in SSA. In the absence of a publically funded healthcare system, these costs are borne almost entirely by individuals - individuals who are among the poorest people in the world. In this context, poor disease prognosis with high morbidity and mortality seem to be the unavoidable outcomes.

\section{Prevention and Care}

Many SSA countries have made significant efforts in initiating and improving care for diabetes and other chronic diseases. A few countries now have established successful national diabetes programmes and guidelines for the management of diabetes. ${ }^{37-39}$ However, in most countries a lot still has to be done. Limited financial resources are a major handicap even where there is the scientific information and the political will is available - both of which are still largely lacking in many SSA countries. It is possible to set up effective diabetes care at the peripheral level using basic resources in terms of personnel and equipment. Nurse-led care including education was shown to be successful in a rural resource-limited setting over an 18-month period. ${ }^{40}$ However, it would be a real challenge to translate such experiments over a larger scale and a much longer period. Work carried out by the International Insulin Foundation ${ }^{41}$ identified key areas to be addressed in the fight against diabetes in SSA, encompassing policy improvement, better organisation and delivery of care and patient education. Whiting et al..$^{42}$ reviewed challenges to the delivery of diabetes care in a few countries that had reported data on diabetes healthcare delivery, and could still identify the following barriers to adequate diabetes care:

- patient attendance is poor (there are many reasons for this and more work is required to explore them);

- consultation times are very short, leaving little or no time for patient education;

- staff levels are inadequate and more use could be made of trained nurses and other health workers;

- staff training is limited, and continuing education or in-service training, especially of lower cadres, is needed;
- complications are not monitored or evaluated in a systematic manner;

- control of blood glucose and blood pressure is very poor;

- referral systems are inadequate;

- patient education is almost non-existent;

- organisation of services is generally poor; and

- better record-keeping will assist improvements in care.

Therefore, even when diabetes care programmes are introduced, a lot will still need to be done to enable them to meet their objective of reducing morbidity and mortality linked to diabetes and its complications. A few of the drawbacks above relate to inadequate healthcare staff levels as well as patient-related factors. Patient education and empowerment in the form of a peers support system should offer some hope of better diabetes control by improving compliance and optimising self care practices.

What if we could prevent diabetes in SSA? Obviously, primary prevention of diabetes has to be the cornerstone of antidiabetes efforts on the continent. This may seem like a tall order; however, on a continent that is impoverished, still fighting infections, has to combine control of undernutrition with rising obesity and has high morbidity and mortality in people who develop diabetes or other chronic diseases, every case of diabetes that is prevented will be a welcome relief. Preventative actions need not be individually tailored to target highrisks individuals given that optimal treatment for people with diabetes is still elusive. Prevention programmes will have to be broad population-based initiatives that will have to be socio-culturally appropriate and involve the local people, ${ }^{43,44}$ providing a heath promotion framework that promotes healthy lifestyles.

The adoption of a World Diabetes Day Resolution by the UN General Assembly ${ }^{45}$ is acknowledgment of the fact that diabetes is a real and imminent threat to social and economic development globally. The UN resolution calls for Member States "to develop national policies for the prevention, treatment and care of diabetes in line with the sustainable development of their healthcare systems, taking into account the internationally agreed development goals, including the Millennium Development Goals". In SSA specifically, the IDF African Region, the World Health Organization (WHO)-AFRO and the African Union have 
jointly issued a Diabetes Declaration and Strategy for Africa. ${ }^{46}$ The declaration is a call to action for governments of African countries and all partners and stakeholders in diabetes to prevent diabetes and related non-communicable diseases and to improve quality of life and reduce morbidity and premature mortality from diabetes (see 'The Diabetes Declaration for Africa - Call to Action', opposite).

\section{Conclusion}

Diabetes is an emerging public health problem in SSA that must be addressed and contained. Its current burden may not confer it a high ranking in the list of health problems of this poor continent. However, it would be counterintuitive to wait for diabetes to move up the list of priorities before appropriate action is taken.

Diabetes research and care in Africa will need to build on the current knowledge and technologies available from other parts of the world. However, a direct transposition may not be a realistic or pragmatic plan since these models will have to be re-moulded into the specificities of the African context. Country-specific data on the burden of the disease are urgently required, together with a clear understanding of the extent of national policies for the prevention and care of diabetes. The Diabetes
Declaration and Strategy for Africa ${ }^{46}$ is a starting point that should be used by the different countries of the region to bring diabetes to the forefront, and at the same time to facilitate the adoption of a structured and holistic approach to establishing a diabetes control programme.

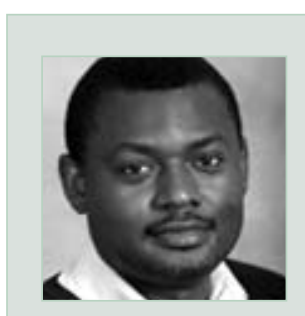

Felix K Assah is a doctoral student at the Medical Research Council Epidemiology Unit in Cambridge. His current research is looking at objectively measured physical activity patterns and metabolic health in urban and rural Cameroon. Dr Assah qualified from the Faculty of Medicine and Biomedical Sciences of the University of Yaoundé I in Cameroon.



Jean-Claude Mbanya is a Professor of Medicine and Endocrinology in the Faculty of Medicine and Biomedical Sciences at the University of Yaoundé I in Cameroon. He is President Elect of the International Diabetes Federation (IDF). Professor Mbanya has worked extensively in Africa and worldwide on advocacy for diabetes. His research has focused on the epidemiology and public health aspects of diabetes in Cameroon and Africa.
1. IDF, Diabetes Atlas, International Diabetes Federation, 2007. Available at: www.eatlas.idf.org/

2. Collier $\mathrm{P}$, The Bottom Billion: Why the Poorest Countries are Failing and What Can Be Done About It, New York: Oxford University Press, 2007

3. Omran AR, The epidemiologic transition: a theory of the epidemiology of population change. 1971, Milbank Q, 2005;83(4):731-57.

4. Majaliwa ES, Elusiyan BE, Adesiyun OO, et al., Type 1 diabetes mellitus in the African population: epidemiology and management challenges, Acta Biomed, 2008;79(3): 255-9.

5. Sobngwi E, Mauvais-Jarvis F, Vexiau P, et al., Diabetes in Africans. Part 2: Ketosis-prone atypical diabetes mellitus, Diabetes Metab, 2002;28(1):5-12.

6. Kalk WJ, Huddle KR, Raal FJ, The age of onset and sex distribution of insulin-dependent diabetes mellitus in Africans in South Africa, Postgrad Med J, 1993;69(813):552-6.

7. Mbanya JC, Kengne AP, Assah F, Diabetes care in Africa, Lancet, 2006;368(9548):1628-9.

8. Mbanya JC, Ngogang J, Salah JN, et al., Prevalence of NIDDM and impaired glucose tolerance in a rural and an urban population in Cameroon, Diabetologia, 1997;40(7): 824-9.

9. Aspray TJ, Mugusi F, Rashid S, et al., Rural and urban differences in diabetes prevalence in Tanzania: the role of obesity, physical inactivity and urban living, Trans R Soc Trop Med Hyg, 2000;94(6):637-44.

10. Sobngwi E, Mbanya JC, Unwin NC, et al., Exposure over the life course to an urban environment and its relation with obesity, diabetes, and hypertension in rural and urban Cameroon, Int J Epidemiol, 2004;33(4):769-76.

11. Levitt NS, Katzenellenbogen JM, Bradshaw D, et al., The prevalence and identification of risk factors for NIDDM in urban Africans in Cape Town, South Africa, Diabetes Care, 1993:16(4):601-7.

12. Sobngwi E, Mbanya JCN, Unwin NC, et al., Physical activity and its relationship with obesity, hypertension and diabetes in urban and rural Cameroon, Int I Obes, 2002;26(7):1009-16.

13. Mbalilaki JA, Hellenius ML, Masesa Z, et al., Physical activity and blood lipids in rural and urban Tanzanians, Nutr Metab Cardiovasc Dis, 2007;17(5):344-8.

14. Levitt NS, Steyn $\mathrm{K}$, Lambert EV, et al., Modifiable risk factors for Type 2 diabetes mellitus in a peri-urban community in South Africa, Diabet Med, 1999;16(11):946-50.

15. World Health Organization, STEPwise approach to chronic disease risk factor surveillance, World Health Organization, 2009. Available at: www.who.int/chp/steps/ reports/en/index.html
16. Bourne $L T$, Lambert EV, Steyn $\mathrm{K}$, Where does the black population of South Africa stand on the nutrition transition?, Public Health Nutr, 2002:5(1A):157-62.

17. Mennen LI, Jackson M, Sharma S, et al., Habitual diet in four populations of African origin: a descriptive paper on nutrient intakes in rural and urban Cameroon, Jamaica and Caribbean migrants in Britain, Public Health Nutr, 2001;4(3):765-72.

18. Abubakari AR, Lauder W, Agyemang $C$, et al., Prevalence and time trends in obesity among adult West African populations: a meta-analysis, Obes Rev, 2008;9(4):297-311.

19. Christensen DL, Eis J, Hansen AW, et al., Obesity and regional fat distribution in Kenyan populations: impact of ethnicity and urbanization, Ann Hum Biol, 2008;35(2):232-49.

20. Fezeu LK, Assah FK, Balkau B, et al., Ten-year changes in central obesity and BMI in rural and urban Cameroon, Obesity (Silver Spring), 2008:16(5):1144-7.

21. Wild S, Roglic G, Green A, et al., Global prevalence of diabetes: estimates for the year 2000 and projections for 2030, Diabetes Care, 2004:27(5):1047-53.

22. Garrett J, Ruel MT, The coexistence of child undernutrition and maternal overweight: prevalence, hypotheses, and programme and policy implications, Matern Child Nutr, 2005;1(3):185-96

23. Health of Populations in Transition (HoPiT) Research Group - Cameroon, Cameroon Burden of Diabetes (CamBoD) Project: Baseline Survey Report, 2004, Yaoundé, Cameroon.

24. Gill G, Gebrekidan A, English P, et al., Diabetic complications and glycaemic control in remote North Africa, QJM, 2008:101(10):793-8.

25. Dehayem YM, Kengne AP, Choukem SP, et al., The spectrum of coma among people with diabetes in Cameroon: an appraisal of the implications and challenges at the Yaounde Central Hospital, Ann Trop Med Parasitol, 2008;102(1):73-8.

26. Rolfe M, Ephraim GG, Lincoln DC, Huddle KR, Hyperosmolar non-ketotic diabetic coma as a cause of emergency hyperglycaemic admission to Baragwanath Hospital, S Afr Med J, 1995;85(3):173-6.

27. Toure NO, Dia KY, Diatta A, et al., Tuberculosis and diabetes, Rev Mal Respir, 2007;24(7):869-75

28. Gill GV, Mbanya JC, Ramaiya KL, Tesfaye S, A sub-Saharan African perspective of diabetes, Diabetologia, 2009;52(1): 8-16.

29. Mbanya JC, Sobngwi E. Diabetes in Africa. Diabetes microvascular and macrovascular disease in Africa, J Cardiovasc Risk, 2003;10(2):97-102.

30. Ogbera OA, Osa E, Edo A, Chukwum E, Common clinical features of diabetic foot ulcers: perspectives from a developing nation, Int J Low Extrem Wounds, 2008:7(2):93-8.

31. Danaei G, Lawes CM, Vander HS, et al., Global and regional mortality from ischaemic heart disease and stroke attributable to higher-than-optimum blood glucose concentration: comparative risk assessment, Lancet, 2006;368(9548):1651-9.

32. Kalk WJ, Joffe BI, Differences in coronary heart disease prevalence and risk factors in African and White patients with type 2 diabetes, Diabetes Res Clin Pract, 2007;77(1): 107-12.

33. Kirigia JM, Sambo HB, Sambo LG, Barry SP, Economic burden of diabetes mellitus in the WHO African region, BMC Int Health Hum Rights, 2009;9(1):6.

34. Kossou-Zinsou SY, Amedegnato MD, Direct cost of hospitalized care in diabetic patients in Lome (Togo), Diabetes Metab, 2001;27(6):709-10.

35. Chale SS, Swai AB, Mujinja PG, McLarty DG, Must diabetes be a fatal disease in Africa? Study of costs of treatment, BMJ, 1992;304(6836):1215-18.

36. Elrayah $\mathrm{H}$, Eltom $\mathrm{M}$, Bedri $\mathrm{A}$, et al., Economic burden on families of childhood type 1 diabetes in urban Sudan, Diabetes Res Clin Pract, 2005;70(2):159-65.

37. Njamnshi A, Hiag AB, Mbanya J-C, From research to policy: the development of a national diabetes programme in Cameroon, Diabetes Voice, 2006;51(3):18-21.

38. Ramaiya K, Tanzania and diabetes-a model for developing countries?, BMJ, 2005;330(7492):679.

39. Daniels A, Biesma R, Otten J, et al., Ambivalence of primary health care professionals towards the South African guidelines for hypertension and diabetes, $S$ Afr Med J, 2000;90(12):1206-11.

40. Gill GV, Price C, Shandu D, et al., An effective system of nurse-led diabetes care in rural Africa, Diabet Med, 2008;25(5):606-11

41. Beran D, Yudkin JS, Diabetes care in sub-Saharan Africa, Lancet, 2006;368(9548):1689-95.

42. Whiting DR, Hayes L, Unwin NC, Diabetes in Africa. Challenges to health care for diabetes in Africa, J Cardiovasc Risk, 2003;10(2):103-10

43. Awah PK, Unwin N, Phillimore P, Cure or control: complying with biomedical regime of diabetes in Cameroon, BMC Health Serv Res, 2008;8:43.

44. Bradley HA, Puoane T, Prevention of hypertension and diabetes in an urban setting in South Africa: participatory action research with community health workers, Ethn Dis, 2007;17(1):49-54.

45. United Nations, World Diabetes Day, A/RES/61/225, 2006

46. IDF Africa, Diabetes Declaration and Strategy for Africa. International Diabetes Federation. Available at: www.idf.org/ 